\title{
Diagnosing Early Ischemic Changes with the Latest-Generation Flat Detector CT: A Comparative Study with Multidetector CT
}

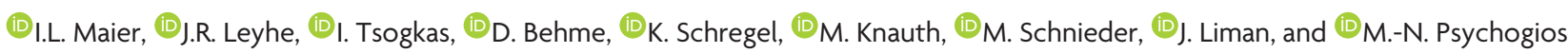

\begin{abstract}
BACKGROUND AND PURPOSE: One-stop management of mechanical thrombectomy-eligible patients with large-vessel occlusion represents an innovative approach in acute stroke treatment. This approach reduces door-to-reperfusion times by omitting multidetector $\mathrm{CT}$, using flat detector $\mathrm{CT}$ as pre-mechanical thrombectomy imaging. The purpose of this study was to compare the diagnostic performance of the latest-generation flat detector CT with multidetector CT.
\end{abstract}

MATERIALS AND METHODS: Prospectively derived data from patients with ischemic stroke with large-vessel occlusion and mechanical thrombectomy were analyzed in this monocentric study. All included patients underwent multidetector CT before referral to our comprehensive stroke center and flat detector $\mathrm{CT}$ in the angiography suite before mechanical thrombectomy. Diagnosis of early ischemic signs, quantified by the ASPECTS, was compared between modalities using cross tables, the Pearson correlation, and Bland-Altman plots. The predictive value of multidetector CT- and flat detector CT-derived ASPECTS for functional outcome was investigated using area under the receiver operating characteristic curve analysis.

RESULTS: Of 25 patients, 24 (96\%) had flat detector CT with sufficient diagnostic quality. Median multidetector CT and flat detector CT ASPECTSs were 7 (interquartile range, 5.5-9 and 4.25-8, respectively) with a mean period of $143.6 \pm 49.5$ minutes between both modalities. The overall sensitivity was $85.1 \%$ and specificity was $83.1 \%$ for flat detector CT ASPECTS compared with multidetector CT ASPECTS as the reference technique. Multidetector CT and flat detector CT ASPECTS were strongly correlated $(r=0.849, P<.001)$ and moderately predicted functional outcome (area under the receiver operating characteristic curve, 0.738; $P=.007$ and $.715 ; P=.069$, respectively).

CONCLUSIONS: Determination of ASPECTS on flat detector CT is feasible, showing no significant difference compared with multidetector CT ASPECTS and a similar predictive value for functional outcome. Our findings support the use of flat detector CT for emergency stroke imaging before mechanical thrombectomy to reduce door-to-groin time.

ABBREVIATIONS: $\mathrm{FDCT}=$ flat detector $\mathrm{CT} ; \mathrm{IQR}=$ interquartile range; $\mathrm{LVO}=$ large-vessel occlusion; $\mathrm{MDCT}=$ multidetector $\mathrm{CT} ; \mathrm{MT}=$ mechanical thrombectomy

I

$\mathrm{n}$ acute major ischemic stroke, the superiority of mechanical thrombectomy (MT) in combination with IV thrombolysis compared with IV thrombolysis alone has been demonstrated in multiple studies. ${ }^{1}$ In patients eligible for MT, symptom-toreperfusion time has been shown to be one of the most impor-

Received October 22, 2017; accepted after revision January 24, 2018.

From the Departments of Neurology (I.L.M., M.S., J.L.) and Neuroradiology (J.R.L., I.T., D.B., K.S., M.K., M.-N.P.), University Medical Center Goettingen, Goettingen, Germany.

This study was supported by the Göttinger Kolleg für Translationale Medizi and the Niedersächsische Ministerium für Wissenschaft und Kultur.

Please address correspondence to Marios-Nikos Psychogios, MD, Department of Neuroradiology, University Medicine Goettingen, Robert-Koch-Str 40, 37075 Goettingen, Germany; e-mail: m.psychogios@med.uni-goettingen.de; @MPeyT1

三 Indicates article with supplemental on-line table.

http://dx.doi.org/10.3174/ajnr.A5595 tant determinants for stroke outcome ${ }^{2}$ and influences stroke mortality. ${ }^{3}$

Thus, the direct transfer of patients with acute cerebral largevessel occlusions (LVOs) to the angiography suite represents an innovative approach to reduce door-to-reperfusion times and consequently to improve functional outcomes. ${ }^{4}$ A recent retrospective analysis comparing patients with direct referral to the angiography suite for MT and patients with prior admission to the emergency department showed significantly reduced symptomto-treatment times and demonstrated the feasibility and safety of this approach. ${ }^{5}$ Rather than omitting the emergency department, another approach to direct angiography suite referral is to skip the multidetector CT (MDCT), performing emergency stroke imaging in the angiography suite with flat detector CT (FDCT) and multiphase flat detector CTA followed by MT without delay. This previously reported approach ${ }^{6,7}$ explicitly addresses the delay 
from MDCT imaging to angiography suite referral, which has been identified as a crucial factor concerning in-hospital delays for the treatment of MT-eligible patients. ${ }^{2}$ However, for this approach, not only intracerebral hemorrhage but also ischemic lesions must be reliably diagnosed by FDCT.

An older study comparing MDCT with FDCT after neurointerventional procedures demonstrated the high specificity and sensitivity of FDCT for the detection of intracerebral hemorrhage and hydrocephalus but could not demonstrate diagnostic sufficiency for the diagnosis of ischemic lesions. ${ }^{8}$ Another, more recent study comparing peri-interventional FDCT with postinterventional MDCT after different neurointerventional procedures again showed high sensitivity and specificity for the detection of intracerebral hemorrhage in different locations. ${ }^{9}$ However, this study was limited in the detection of early ischemic changes on FDCT by a long period between modalities ( 4.3 hours), the use of contrast-/postcontrast agent series, and the use of the ASPECTS as a complete score without the sensitivity/specificity analysis of the predefined brain regions.

The aim of our study was to provide further evidence for the feasibility and reliability of the diagnosis of ischemic changes on FDCT compared with MDCT in patients with acute stroke. Therefore, we compared ASPECTS determined on MDCT and on FDCT performed before MT.

\section{MATERIALS AND METHODS Ethical Approval}

All procedures performed in studies involving human participants were in accordance with the ethical standards of the institutional research committee and with the 1964 Declaration of Helsinki and its later amendments or comparable ethical standards.

\section{Patient Selection}

Clinical and neuroradiologic data were analyzed from a prospectively derived, monocentric data base including neuroradiologic and neurologic information of interventional treatment and clinical outcome. Ethics approval was sought from the ethics committee of our tertiary care center, and all patients or next of kin gave informed written consent for the anonymized use of disease-related data on hospitalization.

Patients who presented to a secondary care hospital with acute major ischemic stroke symptoms where they underwent MDCT and CTA were included. After confirmation of LVO, these patients were referred to our tertiary care center for MT. Patients were transferred directly to the angiography suite, where FDCT and MT were performed ("one-stop management" in acute stroke care). ${ }^{7}$ Clinical data including stroke scores (baseline NIHSS score) and baseline/follow-up mRS scores (at discharge or after 90 days) were determined by a certified stroke neurologist. Favorable functional outcome was defined as an $\mathrm{mRS} \leq 2$ at 90 days or at discharge if the 90-day data were missing.

\section{Data Acquisition}

FDCT was performed with a biplane flat detector angiography system (Artis Q; Siemens, Erlangen, Germany) using the following parameters: 20 seconds of rotation, $200^{\circ}$ total angle with ap- proximately 500 projections, $2 \times 2$ binning, $109 \mathrm{kV}, 1.8 \mu \mathrm{Gy} /$ frame, weighted CT dose index $\sim 60 \mathrm{mGy}$, effective dose $\sim 2.5$ $\mathrm{mSv}$. Initial FDCT projections were then reconstructed on a postprocessing workstation (syngo X Workplace; Siemens) with a Hounsfield unit smooth kernel and DynaCT Clear algorithm (Siemens) to images with a $512 \times 512$ matrix. MDCT before admission was acquired on Somatom Emotion or Somatom Emotion 16 (Siemens) or Optima CT660 scanner (GE Healthcare, Milwaukee, Wisconsin) using a standard brain scan protocol. Raw FDCT and MDCT data were extracted from the PACS of the department, anonymized, reconstructed parallel to the orbitomeatal plane with a slice thickness of $5 \mathrm{~mm}$ and slice distance of $3 \mathrm{~mm}$, and imported for evaluation into the aforementioned postprocessing workstation.

\section{Image Evaluation}

All images were analyzed by 2 experienced neuroradiologists (M.-N.P., with $>5$ years of experience, and K.S., with $>3$ years of experience) and a trained medical student (J.R.L.). Imaging was qualified using the categories "excellent," "slightly compromised," "moderately compromised," and "severely compromised" (not diagnostic). On all MDCT and FDCT scans with sufficient quality, early ischemic signs were determined using the ASPECTS. ${ }^{10}$ The presence of a dense artery sign and intracerebral hemorrhage was noted. FDCT and MDCT images were evaluated with a 4 -week break, to limit recall bias.

\section{Statistical Analysis}

Statistical analysis was performed using the MedCalc statistical package 16.8 (MedCalc Software, Mariakerke, Belgium). Characteristics of all patients are shown as mean \pm SD if normally distributed and as median with interquartile range (IQR), if not. Sensitivity and specificity were determined using cross tables. ASPECTS ratings on FDCT and MDCT were compared using the Pearson correlation and the Bland-Altman plot. Interrater reliability was determined using the Cohen $\kappa$. To assess the predictive value of MDCT and FDCT ASPECTS, we used an area under the receiver operating characteristic curve. Cutoff scores were defined as scores with a maximal Youden index. For all statistical methods, $P$ values $<.05$ were considered significant.

\section{RESULTS}

Twenty-five patients with both MDCT and FDCT before MT were identified (Table 1 ). The mean age was $77.3 \pm 10.3$ years with a median NIHSS score at admission of 17 points (IQR, 14-19 points). Mean symptom-to-groin time was $249.3 \pm 57.1$ minutes with a mean period of $143.6 \pm 49.5$ minutes between MDCT and FDCT. Most patients had an occlusion of either the carotid terminus or the MCA (11 patients [44\%] each), and $88 \%$ of patients were successfully recanalized. Three (12\%) patients were intubated at the time of MT. Median MDCT and FDCT ASPECTSs were 7 (IQR, 5.5-9 and 4.25-8, respectively).

There was no overall difference in the judgment of image quality between MDCT and FDCT (On-line Table). One patient (4.2\%) showed extreme motion artifacts, leading to severely compromised FDCT imaging quality on which the ASPECTS could not be determined. FDCT was judged to have slightly compro- 
Table 1: Baseline characteristics

\begin{tabular}{|c|c|}
\hline Parameter $(n=25)$ & \\
\hline Age (mean) (yr) & $77.3 \pm 10.3$ \\
\hline Sex (male) (\%) & $10(40)$ \\
\hline \multicolumn{2}{|l|}{ Occluded vessel } \\
\hline Proximal ICA (No.) (\%) & $1(4)$ \\
\hline Carotid terminus (No.) (\%) & $11(44)$ \\
\hline M1 (No.) (\%) & $11(44)$ \\
\hline M2 (No.) (\%) & $2(8)$ \\
\hline IV thrombolysis (No.) (\%) & $15(60)$ \\
\hline \multicolumn{2}{|l|}{ Transfer patients ${ }^{\mathrm{a}}$} \\
\hline Drip and ship (No.) (\%) & $14(56)$ \\
\hline Ship and drip (No.) (\%) & $1(4)$ \\
\hline Just ship (No.) (\%) & $10(40)$ \\
\hline General anesthesia at the time of FDCT (No.) (\%) & $3(12)$ \\
\hline Arterial hypertension (No.) (\%) & $19(76)$ \\
\hline Hyperlipoproteinemia (No.) (\%) & $11(44)$ \\
\hline Diabetes mellitus (No.) (\%) & $9(36)$ \\
\hline Atrial fibrillation (No.) (\%) & $8(32)$ \\
\hline Coronary artery disease (No.) (\%) & $9(36)$ \\
\hline Chronic kidney failure (No.) (\%) & $7(28)$ \\
\hline NIHSS score on admission (median) (IQR) & $17(14-19)$ \\
\hline mRS score on admission (median) (IQR) & $5(4-5)$ \\
\hline Successful reperfusion (No.) (\%) & $22(88)$ \\
\hline Symptom-to-groin time (mean) (min) & $249.3 \pm 57.1$ \\
\hline Symptom-to-reperfusion time $(n=22)$ (mean) (min) & $294.6 \pm 66.3$ \\
\hline Door-to-groin time (mean) (min) & $25.2 \pm 8.9$ \\
\hline Door-to-reperfusion time $(n=22)$ (mean) (min) & $77.3 \pm 30$ \\
\hline Symptom-to-MDCT time (mean) (min) & $99.4 \pm 52.6$ \\
\hline Symptom-to-FDCT time (mean) (min) & $234.9 \pm 55.2$ \\
\hline MDCT-to-FDCT time (mean) (min) & $143.6 \pm 49.5$ \\
\hline MDCT ASPECTS (median) (IQR) & $7(5.5-9)$ \\
\hline FDCT ASPECTS (median) (IQR) & $7(4.25-8)$ \\
\hline
\end{tabular}

a Drip and ship: transfer patients with IV thrombolysis started in a peripheral stroke center; ship and drip: transfer patients with IV thrombolysis started in our tertiary stroke center; just ship: transfer patients without IV thrombolysis.

mised image quality in $11(44 \%)$ and moderately compromised image quality in $3(12 \%)$ patients. On FDCT, motion artifacts were visible in $15(60 \%)$ patients; streak-artifacts, in $3(12 \%)$ patients; and metal artifacts, in $1(4 \%)$ patient. The remaining 6 (24\%) patients had no visible artifacts.

Figures 1 and 2 show MDCT and FDCT examples of 2 representative patients, one with ASPECTS of 9 on MDCT and 8 on FDCT and one with ASPECTS of 0 on MDCT and 2 on FDCT (please refer to the figure legends for further details).

As shown in Fig 3, MDCT and FDCT ASPECTS showed a very strong correlation $(r=0.849, P<.001)$. Table 2 gives an overview of all individual ASPECTSs determined on MDCT and FDCT. ASPECTS ratings on FDCT showed a mean difference of 0.8 points (95\% CI, 0.28-1.3 points) compared with MDCT ASPECTS on the Bland-Altmann plot (Fig 4).

Table 3 depicts the sensitivity and specificity of FDCT ASPECTS compared with MDCT ASPECTS. The overall sensitivity was $85.1 \%$ and specificity was $83.2 \%$ for FDCT ASPECTS compared with MDCT as the reference method. When we compared the accuracy of FDCT for the determination of early ischemic signs in every single location, sensitivity and specificity were highest for M2, M4, and the caudate nucleus and lowest for M3, the lentiform nucleus, and internal capsule. The diagnostic accuracy of FDCT to detect a dense media sign was lower compared with detection of early ischemic signs (sensitivity $=72.2 \%$, specificity $=66.7 \%$ ). Interrater agreement for MDCT ASPECTS was

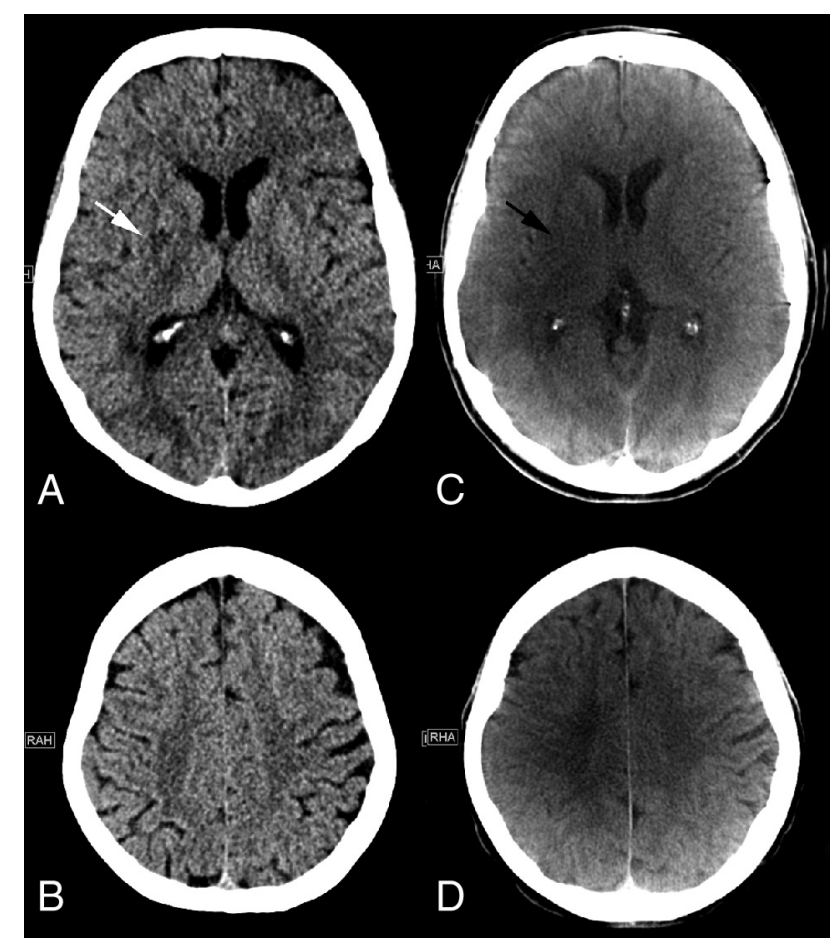

FIG 1. Initial MDCT in the peripheral stroke center shows hypodensity of the right lentiform nucleus (white arrow) and no other early ischemic signs on the ganglionic $(A)$ or supraganglionic $(B)$ level. An ASPECTS of 9 was rated on MDCT images. C, FDCT acquired in our comprehensive stroke center 172 minutes after the initial CT shows hypodensities of the right lentiform nucleus (black arrow) and insula. No early ischemic signs were detected on the supraganglionic level, resulting in an ASPECTS of 8.

excellent between the 2 neuroradiologists $(\kappa=0.942, P<.001)$ and between the most experienced neuroradiologist and the trained medical student $(\kappa=0.843, P<.001)$. For FDCT ASPECTS, the interrater agreement was also excellent between the 2 neuroradiologists $(\kappa=0.865, P<.001)$ and good between the most experienced neuroradiologist and the trained medical student $(\kappa=0.713, P<.001)$.

Eight patients $(33.3 \%)$ had a follow-up $\mathrm{mRS}$ of $\leq 2$. Figure 5 shows the receiver operating characteristic curves of both the MDCT and FDCT ASPECTS for the prediction of favorable functional outcome. The predictive value of both MDCT and FDCT ASPECTS was moderate (area under the receiver operating characteristic curve MDCT ASPECTS $=0.738 ; P=.007$; FDCT ASPECTS $=0.715 ; P=.069$ ). The comparison of the predictive values of both scores revealed no statistically significant difference $(P=.778)$.

\section{DISCUSSION}

Innovative approaches to reduce door-to-groin times have been proposed recently for patients with LVO. ${ }^{5,6,9,11}$ These approaches include the direct referral of MT-eligible patients to the angiography suite with a variation of imaging or no imaging proposed by the various authors. We have recently published an innovative one-stop-management protocol, which includes both nonconstrast imaging and multiphase CTA (acquired in the angiography suite). Omitting MDCT seems plausible because it has been shown that among 1000 patients with successful MT, for every 15 


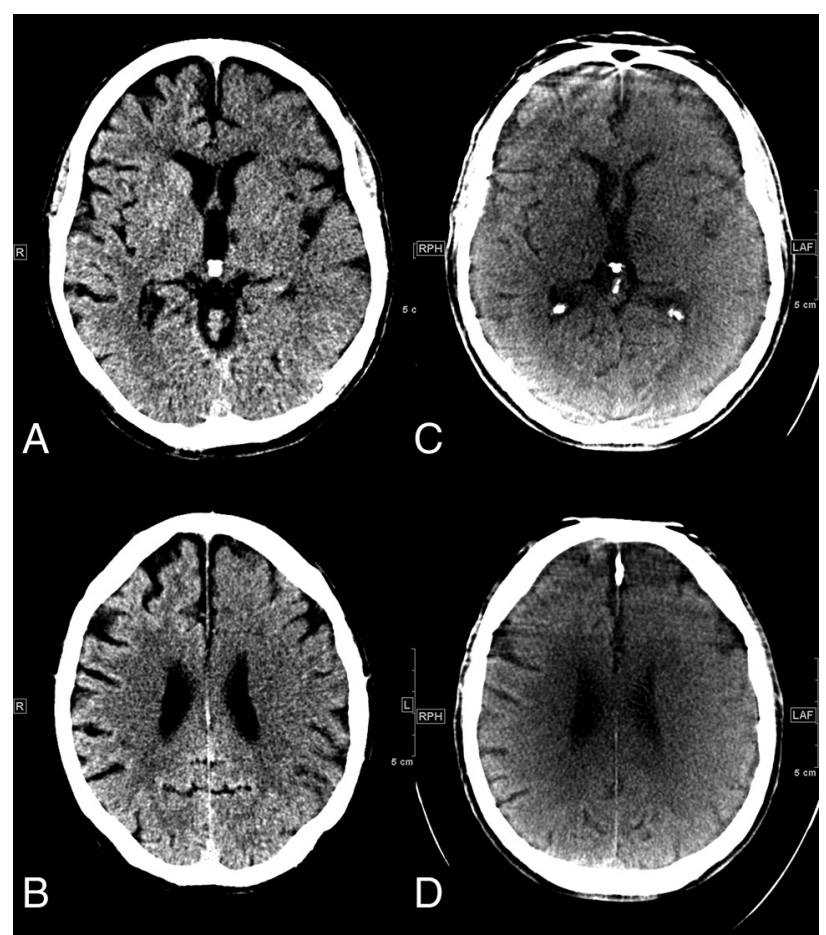

FIG 2. A, Initial MDCT in the peripheral stroke center shows hypodensities of the left striatum, insula, internal capsule, M1, M2, and $\mathrm{M} 3$ segments. $B$, In the supraganglionic levels, we observe early signs in the M4, M5, and M6 segments, resulting in an ASPECTS of $0 . C$ and D, FDCT acquired in our comprehensive stroke center 94 minutes after the initial CT shows early ischemic signs in the left anterior cerebral artery and MCA territories, resulting in an ASPECTS of 2. The M3 and M6 segments are not classified as ischemic on FDCT images.

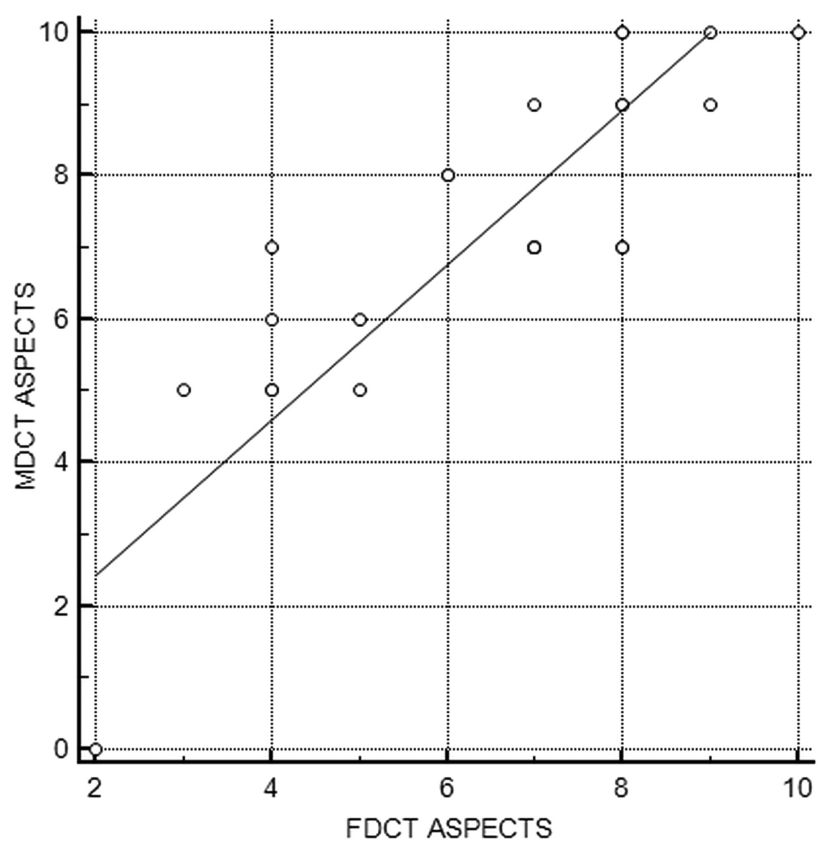

FIG 3. Pearson correlation between MDCT and FDCT ASPECTS.

minutes of faster door-to-reperfusion time, 39 patients would be less disabled at 3-month follow-up, including 25 patients achieving an mRS of $0-2 .^{2}$ In this context, the Highly Effective Reperfusion evaluated in Multiple Endovascular Stroke trials (HERMES) collaborators reported that only $4 \%$ of the patients
Table 2: Overview of all individual ASPECTS determined on MDCT and FDCT

\begin{tabular}{lcc}
\hline & \multicolumn{2}{c}{ ASPECTS } \\
\cline { 2 - 3 } Patient No. & MDCT & FDCT \\
\hline 1 & 10 & 8 \\
2 & 9 & 8 \\
3 & 7 & 7 \\
4 & 10 & 10 \\
5 & 10 & 8 \\
6 & 7 & 8 \\
7 & 7 & 7 \\
8 & 8 & 6 \\
9 & 9 & 7 \\
10 & 5 & 5 \\
11 & 3 & NA \\
12 & 6 & 5 \\
13 & 9 & 8 \\
14 & 9 & 9 \\
15 & 9 & 4 \\
16 & 5 & 4 \\
17 & 7 & 7 \\
18 & 7 & 2 \\
19 & 7 & 3 \\
20 & 0 & 7 \\
21 & 5 & 6 \\
22 & 7 & 4 \\
23 & 5 & 9 \\
24 & 6 & 4 \\
25 & 10 & 8 \\
\hline
\end{tabular}

Note:-NA indicates not applicable.

${ }^{a}$ Patient 11 had an FDCT with severely compromised (nondiagnostic) imaging quality.

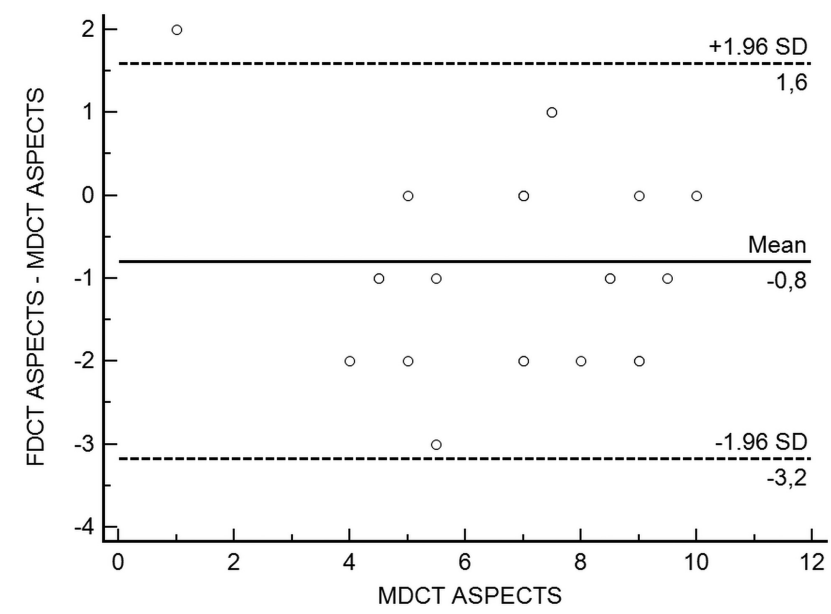

FIG 4. Bland-Altman plot of MDCT and FDCT ASPECTS.

included in the most recent large thrombectomy trials had an MDCT-to-groin puncture time of $\leq 30$ minutes, which represents the latest recommendation of the Society of NeuroInterventional Surgery. ${ }^{2,12}$

However, the approach to perform stroke imaging directly in the angiography suite requires comparable diagnostic accuracy of FDCT and MDCT as the reference method. This includes comparable sensitivity to detect intracerebral hemorrhage, vessel occlusion, and collateral status as well as ischemic changes. For the latter, it can be questioned whether detection of early ischemic signs on initial FDCT is necessary at all because there is evidence that patients with low ASPECTS also benefit from MT. ${ }^{13}$ However, the pre-MT ASPECTS can be very important for the treating 


\begin{tabular}{|c|c|c|c|c|c|}
\hline & $\begin{array}{l}\text { Early Ischemic } \\
\text { Signs on MDCT }\end{array}$ & $\begin{array}{l}\text { Early Ischemic } \\
\text { Signs on FDCT }\end{array}$ & $\begin{array}{l}\text { Early Ischemic Signs } \\
\text { on MDCT and FDCT }\end{array}$ & Sensitivity & Specificity \\
\hline Ml (No.) (\%) & $2(8.3)$ & $3(12.5)$ & $1(4.2)$ & 50 & 90.1 \\
\hline M2 (No.) (\%) & $2(8.3)$ & $7(29.2)$ & $2(8.3)$ & 100 & 77.3 \\
\hline M3 (No.) (\%) & $1(4.2)$ & $1(4.2)$ & $0(0)$ & 0 & 95.8 \\
\hline C (No.) (\%) & $14(58.3)$ & $14(58.3)$ & $13(54.2)$ & 92.9 & 90 \\
\hline L (No.) (\%) & $15(62.5)$ & $21(58.3)$ & $14(58.3)$ & 93.2 & 22.2 \\
\hline IC (No.) (\%) & $3(12.5)^{\prime}$ & 4 (16.7) & $1(4.2)^{\prime}$ & 33.3 & 85.7 \\
\hline I (No.) (\%) & $18(75)$ & 22 (91.7) & $18(75)$ & 100 & 33.3 \\
\hline M4 (No.) (\%) & $5(20.8)$ & $8(33.3)$ & $5(20.8)$ & 100 & 84.2 \\
\hline M5 (No.) (\%) & $4(16.7)$ & $6(25)$ & $3(12.5)$ & 75 & 85 \\
\hline M6 (No.) (\%) & $3(12.5)$ & $0(0)$ & $0(0)$ & NA & NA \\
\hline Overall (No.) (\% of 240 locations) & $67(27.9)$ & $86(35.8)$ & $57(23.8)$ & 85.1 & 83.2 \\
\hline Dense media sign (No. positive) (\%) & $18(75)$ & $15(62.5)$ & $13(54.2)$ & 72.2 & 66.7 \\
\hline
\end{tabular}

Note:-Anatomic regions according to the Alberta Stroke Program Early CT Score: M1-M6 indicate cortical regions of the medial cerebral artery territory; C, caudate nucleus; L, lentiform nucleus; IC, internal capsule; I, insula; NA, not applicable.

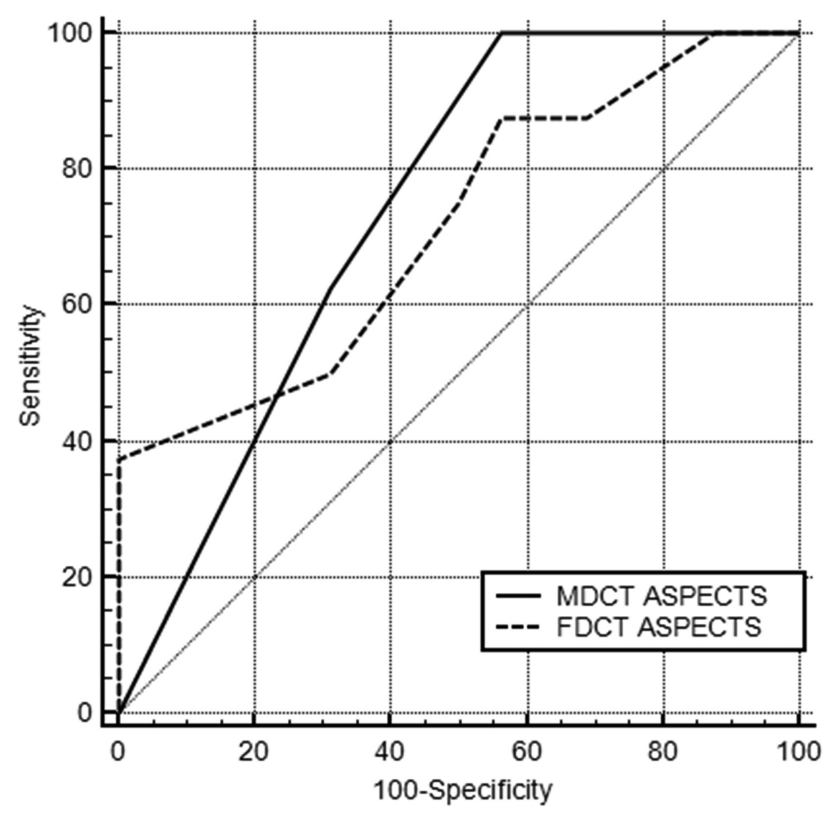

FIG 5. Area under the receiver operating curve analysis for the predictive value of MDCT and FDCT ASPECTS for favorable functional outcome $(m R S \leq 2)$.

neurointerventionalist in patients with wake-up strokes or unknown clinical onset as well as in scenarios in which intervention is prolonged and termination of the MT has to be discussed after multiple thrombectomy maneuvers.

There is growing evidence that FDCT can be reliably used to exclude intracerebral hemorrhage ${ }^{9,14,15}$ and that the use of timeresolved flat detector CTA and flat detector CTP is feasible, showing sufficient reliability for decision-making. ${ }^{16,17}$ One of the most recent studies addressing the reliability of FDCT in acute stroke by Eckert et $\mathrm{al}^{15}$ also reported data on the reliability of detecting ischemic changes on FDCT. This study reported poor sensitivity for FDCT to detect early ischemic signs compared with post-MT MDCT. However, the authors of this study evaluated FDCT images acquired with an older angiography system (Axiom Artis dBA; Siemens) and did not use ASPECTS as a systematic evaluation method to search for early ischemic changes, and only 4 $(12.5 \%)$ of the 32 patients with ischemic stroke included in this study had early ischemic changes on the initial MDCT. ${ }^{15}$ In contrast, we used a latest-generation angiography suite with 16-bit readout, which allows better contrast resolution compared with the older systems. Additionally, $20(83.3 \%)$ of the 24 patients included in our study had early ischemic signs on initial MDCT (ASPECTS $<10$ ), which renders our cohort more eligible for the comparison of ischemic signs detected on MDCT versus FDCT. Moreover, Eckert et $\mathrm{al}^{15}$ also included patients with vertebral and basilar artery occlusions, predominantly causing infratentorial ischemia, for which the diagnostic accuracy of FDCT is generally lower compared with the supratentorial region. ${ }^{9}$ In contrast to the study by Eckert et al, our results are in line with findings from a previous study by Leyhe et $\mathrm{al}^{9}{ }^{9}$ which showed high sensitivity and specificity for detecting large ischemic changes (ASPECTS $\leq 5)$ on FDCT compared with MDCT.

In our study, FDCT ASPECTS was 0.8 points lower compared with MDCT. This difference could be explained by the delay between both modalities, in which ischemia could have developed in the context of persistent LVO during the transfer from the secondary care hospital to our comprehensive stroke center. This difference is in agreement with findings from a study by Sun et $\mathrm{al},{ }^{18}$ which showed an average ASPECTS decline of 1 point if the patient was transferred from another hospital for MT. In the study by Leyhe et $\mathrm{al}^{9}{ }^{9}$ a mean ASPECTS difference between peri-interventional FDCT and postinterventional MDCT of 0.5 points was described. The higher difference found in the present study could be explained by ongoing ischemia during the period between MDCT and pre-MT FDCT, while ASPECTS comparison after successful MT (as done by Leyhe et al) is likely to be more stable. ${ }^{9}$

Besides the difference in ASPECTS between both modalities caused by the delay between MDCT and FDCT, increased sensitivity and specificity for FDCT ASPECTS could have been expected with a higher number of patients with general anesthesia during MT. Motion artifacts are a common problem in FDCT imaging because they can affect multiple slices and can occur more often in imaging techniques with prolonged image-acquisition times. Thus, in the latest flat detector systems, image acquisition takes 20 seconds, which increases the risk for motion artifacts. ${ }^{9,19}$ This is a common problem, especially in the acute setting of ischemic stroke, in which patients are often not cooperative due to aphasia or confusion. In our study, most patients underwent 
MT without general anesthesia, which explains the high percentage of motion artifacts despite adequate positioning and head fixation, which have been described in detail elsewhere. ${ }^{9}$ These artifacts could also have been the major reason for the lower sensitivity and specificity of FDCT to detect a dense media sign because it is seen in only a small, deep brain region on a few slices. Eckert et $\mathrm{al}^{15}$ reported a comparable rate of motion artifacts but a significantly lower sensitivity to detect a dense vessel sign (a hyperdense media sign was present in 7 cases, and none were detected on FDCT). These findings underline the diagnostic limitations for the detection of dense media signs and urge combined use with flat detector CTA to reliably diagnose LVO. Additionally, the development of a faster protocol with identical contrast resolution would reduce the presence of motion artifacts.

Our findings must be interpreted considering several limitations of our study. We report retrospective, single-center data, which cannot be applied to other centers using different FDCT systems and imaging-reconstruction methods. In addition, our sample size is small, and ASPECTS raters were not blinded to the ischemic side.

\section{CONCLUSIONS}

Our study provides evidence for the feasibility and reliability of ASPECTS determination on FDCT in MT-eligible patients. Given the use of optimized, latest-generation flat detectors and optimal positioning strategies, these findings add to the one-stop approach to acute stroke care, in which emergency stroke imaging is performed in the angiography suite directly before MT, aiming to reduce door-to-groin times.

Disclosures: Ilko L. Maier—RELATED: Niedersächsische Ministerium für Wissenschaft und Kultur, Comments: The first author of this study received a grant from the Göttinger Kolleg für Translationale Medizin, which was supported by the Niedersächsische Ministerium für Wissenschaft und Kultur. Daniel Behme-UNRELATED: Payment for Lectures Including Service on Speakers Bureaus: Penumbra Deutschen Gesellschaft für Neuroradiologie e.V. (DGNR), Acandis DGNR Cologne 2017. Michael Knauth—RELATED: Grant: Siemens, Comments: research cooperation*; Consulting Fee or Honorarium: Siemens, Comments: speakers bureau; Support for Travel to Meetings for the Study or Other Purposes: Siemens, Comments: research cooperation*; UNRELATED: Consultancy: Acandis; Payment for Development of Educational Presentations: Bayer HealthCare, Comments: speakers bureau; Stock/Stock Options: Siemens, Comments: shareholder. Jan Liman-UNRELATED: Payment for Lectures Including Service on Speakers Bureaus: Stryker. Marios-Nikos Psychogios-RELATED: Grant: Siemens*; UNRELATED: Consultancy: Siemens, Stryker, Acandis, Penumbra; Payment for Development of Educational Presentations: phenox. *Money paid to the institution.

\section{REFERENCES}

1. Goyal M, Menon BK, van Zwam WH, et al; HERMES collaborators. Endovascular thrombectomy after large-vessel ischaemic stroke: a meta-analysis of individual patient data from five randomised trials. Lancet 2016;387:1723-31 CrossRef Medline

2. Saver JL, Goyal M, van der Lugt A, et al; HERMES Collaborators. Time to treatment with endovascular thrombectomy and outcomes from ischemic stroke: a meta-analysis. JAMA 2016;316:1279-88 CrossRef Medline

3. Rinaldo L, Brinjikji W, McCutcheon BA, et al. Hospital transfer associated with increased mortality after endovascular revascularization for acute ischemic stroke. J Neurointerv Surg 2017;9:1166-72 CrossRef Medline
4. Hung SC, Lin CJ, Guo WY, et al. Toward the era of a one-stop imaging service using an angiography suite for neurovascular disorders. Biomed Res Int 2013;2013:873614 CrossRef Medline

5. Jadhav AP, Kenmuir CL, Aghaebrahim A, et al. Interfacility transfer directly to the neuroangiography suite in acute ischemic stroke patients undergoing thrombectomy. Stroke 2017;48:1884-89 CrossRef Medline

6. Ribo M, Boned S, Rubiera M, et al. Direct transfer to angiosuite to reduce door-to-puncture time in thrombectomy for acute stroke. J Neurointerv Surg 2018;10:221-24 CrossRef Medline

7. Psychogios MN, Bähr M, Liman J, et al. One stop management in acute stroke: first mothership patient transported directly to the angiography suite. Clin Neuroradiol 2017;27:389-91 CrossRef Medline

8. Psychogios MN, Buhk JH, Schramm P, et al. Feasibility of angiographic CT in peri-interventional diagnostic imaging: a comparative study with multidetector CT. AJNR Am J Neuroradiol 2010;31: 1226-31 CrossRef Medline

9. Leyhe JR, Tsogkas I, Hesse AC, et al. Latest generation of flat detector CT as a peri-interventional diagnostic tool: a comparative study with multidetector CT. J Neurointerv Surg 2017;9:1253-57 CrossRef Medline

10. Pexman JH, Barber PA, Hill MD, et al. Use of the Alberta Stroke Program Early CT Score (ASPECTS) for assessing CT scans in patients with acute stroke. AJNR Am J Neuroradiol 2001;22:1534-42 Medline

11. Psychogios MN, Behme D, Schregel K, et al. One-stop management of acute stroke patients: minimizing door-to-reperfusion times. Stroke 2017;48:3152-55 CrossRef Medline

12. McTaggart RA, Ansari SA, Goyal M, et al; Standards and Guidelines Committee of the Society of NeuroInterventional Surgery (SNIS). Initial hospital management of patients with emergent large vessel occlusion (ELVO): report of the standards and guidelines committee of the Society of Neurointerventional Surgery. J Neurointerv Surg 2017;9:316-23 CrossRef Medline

13. Yoo AJ, Berkhemer OA, Fransen PSS, et al; MR CLEAN investigators. Effect of baseline Alberta Stroke Program Early CT Score on safety and efficacy of intra-arterial treatment: a subgroup analysis of a randomised phase 3 trial (MR CLEAN). Lancet Neurol 2016;15: 685-94 CrossRef Medline

14. Frölich AM, Buhk JH, Fiehler J, et al. Voxel-based sensitivity of flatpanel CT for the detection of intracranial hemorrhage: comparison to multi-detector CT. PLoS One 2016;11:e165794 CrossRef Medline

15. Eckert M, Gölitz P, Lücking H, et al. Optimized flat-detector CT in stroke imaging: ready for first-line use? Cerebrovasc Dis 2017;43: 9-16 CrossRef Medline

16. Niu K, Yang P, Wu Y, et al. C-arm conebeam CT perfusion imaging in the angiographic suite: a comparison with multidetector CT perfusion imaging. AJNR Am J Neuroradiol 2016;37:1303-09 CrossRef Medline

17. Yang P, Niu K, Wu Y, et al. Evaluation of collaterals and clot burden using time-resolved C-arm conebeam CT angiography in the angiography suite: a feasibility study. AJNR Am J Neuroradiol 2017;38: 747-52 CrossRef Medline

18. Sun CH, Connelly K, Nogueira RG, et al. ASPECTS decay during inter-facility transfer predicts patient outcomes in endovascular reperfusion for ischemic stroke: a unique assessment of dynamic physiologic change over time. J Neurointerv Surg 2015;7:22-26 CrossRef Medline

19. Struffert T, Eyupoglu IY, Huttner HB, et al. Clinical evaluation of flat-panel detector compared with multislice computed tomography in 65 patients with acute intracranial hemorrhage: initial results. Clinical article. J Neurosurg 2010;113:901-07 CrossRef Medline 